University of Nebraska - Lincoln

DigitalCommons@University of Nebraska - Lincoln

February 1970

\title{
Fermi Surface of AuSb2. I. High-Field Galvanomagnetic Effects
}

\author{
J. Ahn \\ University of Nebraska - Lincoln \\ David J. Sellmyer \\ University of Nebraska-Lincoln, dsellmyer@unl.edu
}

Follow this and additional works at: https://digitalcommons.unl.edu/physicssellmyer

Part of the Physics Commons

Ahn, J. and Sellmyer, David J., "Fermi Surface of AuSb2. I. High-Field Galvanomagnetic Effects" (1970).

David Sellmyer Publications. 182.

https://digitalcommons.unl.edu/physicssellmyer/182

This Article is brought to you for free and open access by the Research Papers in Physics and Astronomy at DigitalCommons@University of Nebraska - Lincoln. It has been accepted for inclusion in David Sellmyer Publications by an authorized administrator of DigitalCommons@University of Nebraska - Lincoln. 


\title{
Physical RevieW B SOLID STATE
}

\section{Fermi Surface of $\mathrm{AuSb}_{2}$. I. High-Field Galvanomagnetic Effects*}

\author{
J. Ahn† AND D. J. Sellmyer \\ Center for Materials Science and Engineering, Massachusetts Institute of Technology, Cambridge, Massachusetts 02139
}

(Received 30 April 1969; revised manuscript received 6 October 1969)

\begin{abstract}
High-field galvanomagnetic effects have been investigated in the pyrite structure metallic compound $\mathrm{AuSb}_{2}$ in fields up to $150 \mathrm{kG}$. The resistance ratios of the samples $\left[\rho\left(295^{\circ} \mathrm{K}\right) / \rho\left(4.2^{\circ} \mathrm{K}\right)\right]$ were as high as 520 . The results indicate that $\mathrm{AuSb}_{2}$ is a compensated metal and that its Fermi surface supports open orbits in $\langle 100\rangle,\langle 110\rangle$, and $\langle 112\rangle$ directions. At fields higher than about $50 \mathrm{kG}$, the tendency toward saturation caused by the open orbits changes to a field dependence approaching quadratic, and rather large-amplitude quantum oscillations in the magnetoresistance appear. This behavior is explicable in terms of a loss of the open orbits in the highest fields as a result of magnetic breakdown. An approximate calculation based on the theory of magnetic breakdown gives a value of $85 \mathrm{meV}$ for the size of the energy gaps involved in the breakdown.
\end{abstract}

\section{INTRODUCTION}

$I^{\mathrm{N}}$ $\mathrm{N}$ the last decade, our knowledge of the Fermi surface of metals has broadened enormously through the application of a variety of experimental techniques such as the de Haas-van Alphen effect (dHvA) and high-field magnetoresistance (MR). In the past several years, with the realization that sufficiently pure samples could be prepared, these techniques have been extended to certain metallic compounds and ordered alloys. The number of compounds investigated to date is not large, but as more of them are investigated, it is becoming increasingly clear that the nearly free-electron (NFE) or single-orthogonalized-plane-wave model is a useful first approximation to the Fermi surface of many of these metals. Some examples of metallic compounds or ordered alloys whose Fermi surfaces are NFE-like are the $\mathrm{CsCl}$ structure compounds $\mathrm{CuZn},{ }^{1,2} \mathrm{AgZn},{ }^{1}$ $\mathrm{PdIn}^{1}$; the fluorite structure compounds $\mathrm{AuAl}_{2}, \mathrm{AuGa}_{2}$, $\mathrm{AuIn}_{2}{ }^{3-5}$; and the NiAs structure compound AuSn. ${ }^{6,7}$

* Research supported by the Advanced Research Projects Agency and performed in part at the Francis Bitter National Magnet Laboratory which is supported by the U. S. Air Force Office of Scientific Research.

† IBM Graduate Fellow. Present address: IBM, East Fishkill Facility, Hopewell Junction, N. Y.

1 J.-P. Jan, W. B. Pearson, and Y. Saito, Proc. Roy. Soc. (London) A297, 275 (1967).

${ }^{2}$ D. J. Sellmyer, J. Ahn, and J.-P. Jan, Phys. Rev. 161, 618 (1967).

3 J.-P. Jan, W. B. Pearson, Y. Saito, M. Springford, and I. M. Templeton, Phil. Mag. 12, 1271 (1965).

${ }^{4}$ J. T. Longo, P. A. Schroeder, and D. J. Sellmyer, Phys. Letters 25A, 747 (1967); Phys. Rev. 182, 658 (1969).

5 J. A. Rayne, Phys. Letters 7, 114 (1963).

6 D. J. Sellmyer and P. A. Schroeder, Phys. Letters 16, 100

(1963) ; D. J. Sellmyer, J. Phys. Chem. Solids 30, 2371 (1969).

${ }^{7}$ G. J. Edwards, M. Springford, and Y. Saito, J. Phys. Chem. Solids 30, 2527 (1969).
There appear to be three major problems concerning Fermi-surface determinations for compounds: (a) preparation of samples of high-enough structural perfection, (b) first-principles energy-band calculations are difficult either because of the large number of atoms per primitive cell or because of the complex nature of the crystal structure which makes the choice of lattice potential very difficult, and (c) the electron concentration in the conduction band of metallic compounds is, in general, an unknown quantity. With regard to (c), the usual practice has been to assume that the valence is determined by the column of the Periodic Table to which each constituent atom belongs and that all the valence electrons go into the conduction bands of the compound.

The object of this research has been to extend Fermisurface investigations of metallic compounds to crystal structures other than the ones listed above, and to further test the validity of the NFE model. Among the several candidate compounds, $\mathrm{AuSb}_{2}$ was chosen because (a) it has the pyrite structure which is somewhat more complicated than compounds previously investigated and therefore the chemical binding forces may be more complicated, (b) it has been investigated by ordinary transport properties ${ }^{8,9}$ and, to a limited extent, by the dHvA effect, ${ }^{10}$ and single crystals of $\mathrm{AuSb}_{2}$ could be grown with quite high purity.

We have investigated three types of phenomena in $\mathrm{AuSb}_{2}$ : high-field galvanomagnetic effects, quantum

${ }^{8}$ W. B. Pearson, Can. J. Phys. 42, 519 (1964).

${ }^{9}$ M. P. Mathur, R. C. Miller, and D. H. Damon, Bull. Am. Phys. Soc. 12, 1040 (1967); P. R. Emtage, D. H. Damon, M. P. Mathur, and R. C. Miller, Phys. Rev. 180, 797 (1969).

${ }_{10}$ A. Beck, J.-P. Jan, W. B. Pearson, and I. M. Templeton, Phil. Mag. 8, 351 (1963).

\section{1273}


TABLE I. Summary of transverse MR in the high-field limit. ${ }^{a}$

\begin{tabular}{|c|c|c|c|c|}
\hline Type & State of compensation & Nature of orbits & MR behavior & Hall field ${ }^{b}$ \\
\hline $\mathrm{I}$ & uncompensated & all closed & $\sim B_{0}^{0}$ & $\Omega \quad B$ \\
\hline II & compensated & all closed & $\sim B^{2}$ & $n_{e}-n_{h} e c$ \\
\hline III & either & $\begin{array}{l}\text { open orbit in } \mathrm{k} \text { space making } \\
\text { angle } \gamma \text { with } \mathrm{J}\end{array}$ & $\sim B^{2} \cos ^{2} \gamma$ & $\sim B$ \\
\hline IV & either & $\begin{array}{l}\text { non-intersecting open orbits in } \\
2 \text { different directions }\end{array}$ & $\sim B^{0}$ & $\sim B^{-1}$ \\
\hline \multirow{2}{*}{$\mathrm{V}$} & \multirow{2}{*}{ either } & \multirow{2}{*}{ singular-field direction } & \multirow{2}{*}{$\sim B^{0}$} & $-\Omega \quad B$ \\
\hline & & & & $n_{A} \pm \Delta n e c$ \\
\hline
\end{tabular}

a After Ref. 15, to which reference should be made for definitions of singular-field directions and $\Delta n$.

b That is, electric field per unit current density. $\Omega$ is the volume of a primitive cell of the lattice.

oscillations in the magnetoresistance, and the dHvA effect. The scope of the present paper is limited to a discussion of the nonoscillatory part of the MR and the Hall coefficient in the high-field region and their implications concerning topological properties of the Fermi surface of $\mathrm{AuSb}_{2}$. The oscillatory $\mathrm{MR}$ and $\mathrm{dHvA}$ effect are discussed in the second paper of this series, ${ }^{11}$ hereafter referred to as II. A discussion of the NFE Fermi-surface model for $\mathrm{AuSb}_{2}$ is deferred to II where the predictions of this model are compared with the results of the MR and quantum oscillatory experiments.

\section{THEORY}

\section{A. High-Field Galvanomagnetic Effects}

Lifshitz, Azbel, and Kaganov ${ }^{12}$ and Lifshitz and Peschanskii ${ }^{13,14}$ showed that an investigation of the low-temperature galvanomagnetic properties of a metal can, in the case of high magnetic fields and sample purities, yield information about the topology of the Fermi surface of the metal. Recently, Fawcett has given a comprehensive review of the theoretical and experimental situation with regard to high-field galvanomagnetism in pure metals. ${ }^{15}$

There are two important physical situations which affect the resistivity tensor in the high-field limit which is defined by the condition $\left.\left\langle\omega_{c} \tau\right\rangle_{\text {av }}\right\rangle 1$, where $\omega_{c}$ is the cyclotron frequency and $\tau$ is the electronic relaxation time. These are the state of compensation of the metal and the presence of open orbits. The state of compensation of a metal is defined as follows. ${ }^{2,15}$ Let $n_{e}\left(n_{h}\right)$ be the number of occupied (empty) states per primitive

\footnotetext{
${ }^{11} \mathrm{~J}$. Ahn, and D. J. Sellmyer, following paper, Phys. Rev. B 1, 1285 (1970).

${ }^{12}$ I. M. Lifshitz, M. I. Azbel, and M. I. Kaganov, Zh. Eksperim. i, Teor. Fiz. 31, 63 (1956) [English transl.: Soviet Phys.-JETP 4, 41 (1967)].

${ }_{13}$ I. M. Lifshitz and V. G. Peschanskii, Zh. Eksperim. i Teor Fiz. 35, 1251 (1958) [English transl.: Soviet Phys.-JETP 8, $875(1959)]$.

${ }_{14}$ I. M. Lifshitz and V. G. Peschanskii. Zh. Eksperim. i Teor. Fiz. 38, 188 (1960) [English transl.: Soviet Phys.-JETP 11, 137 (1960)].

${ }^{15}$ E. Fawcett, Advan. Phys. 13, 139 (1964).
}

cell in partially filled zones and let $n_{A} \equiv n_{h}-n_{e}$. A given metal is said to be compensated if $n_{A}=0$ and uncompensated if $n_{A} \neq 0$. The reason for the importance of the state of compensation is that the field dependence of the conductivity tensor depends on the value of $n_{A}$ which in turn is related to the Hall coefficient. For future reference in discussing the results, the field and orientation dependence of the transverse MR and Hall field under various conditions is summarized in Table I. The important feature of the theory is the major difference in the predicted field dependence of the MR depending on $n_{A}$. If $n_{A} \neq 0$, the MR saturates if $\mathbf{B}$ is in a general direction, but becomes quadratic in $\mathbf{B}$ when there exist open orbits normal to $\mathbf{B}$ (and $\mathbf{J}$ is not perpendicular to the open-orbit direction). If $n_{A}=0$, on the other hand, the MR increases quadratically in B for a general field direction but saturates only when there exist open orbits normal to both current and field directions. This means that, for $n_{A}=0$, the current axes must be chosen so that they are nearly normal to various open-orbit directions if they are to be observed.

\section{B. Magnetic-Breakdown Phenomena}

The theory discussed above assumed that there were no interband transitions. However, Cohen and Falicov ${ }^{16}$ and Blount ${ }^{17}$ have shown that in metals in which an energy gap $\epsilon_{g}$ is small enough to satisfy

$$
\hbar \omega_{c} \gtrsim K \epsilon_{g}^{2} / \epsilon_{F}
$$

where $\epsilon_{F}$ is the Fermi energy and $K$ is a numerical factor of order unity, the electrons have a finite probability of making transitions between the two energy bands separated by $\epsilon_{g}$. Such small energy gaps often occur at points in $\mathbf{k}$ space when certain degeneracies are removed by weak interactions such as the lattice potential or the spin-orbit interaction. The value

$$
B_{c}=K \epsilon_{g}^{2} m_{c} c / e \hbar \epsilon_{F}
$$

is called the critical field for magnetic breakdown and

${ }^{16}$ M. H. Cohen and L. M. Falicov, Phys. Rev. Letters 7, 231 (1961).

${ }_{17}$ E. I. Blount, Phys. Rev. 126, 1636 (1962). 
is related to the interband transition probability $P$ by ${ }^{17}$

$$
P=e^{-B_{c} / B} .
$$

Magnetic breakdown can modify the behavior of the transverse MR summarized in Table I. As the highfield MR effects are sensitive to the topology of the Fermi surface, it is clear that any change in the topology due to breakdown will be reflected in the high-field MR measurements. A semiclassical approach to this problem was investigated by Falicov and Sievert, ${ }^{18}$ who considered many cases of physical interest. While the phenomenon of magnetic breakdown is interesting in itself, it can obscure the high-field effects when employed to explore the "intrinsic" Fermi surface of a metal. Because of this, considerable care must be exercised in interpreting high-field results when breakdown occurs. The subject of magnetic-breakdown metals has been reviewed recently by Stark and Falicov..$^{19}$

\section{EXPERIMENTAL AND OTHER DETAILS}

\section{A. Crystal Structure}

The pyrite crystal structure of $\mathrm{AuSb}_{2}$ is shown in Fig. 1. Each gold atom is surrounded octahedrally by six antimony atoms and each antimony is surrounded tetrahedrally by one antimony atom and three gold atoms. The cubic cell shown in Fig. 1 contains four units of $\mathrm{AuSb}_{2}$. Since the primitive cell of the structure has the simple cubic Bravais lattice, it follows that the Brillouin zone is also simple cubic.

A consideration of Fig. 1 and the space group $T_{h}{ }^{6}$ shows that the pyrite structure does not have fourfold rotation symmetry about the $\langle 100\rangle$ directions. ${ }^{20}$ Thus, there are two types of $\{100\}$ planes, here denoted as $\{100\}_{i}$ where $i=\alpha$ or $\beta$, which can be distinguished by careful inspection of the Laue photographs taken with a $\{100\}$ plane normal to the x-ray beam..$^{21}$ Two of the mutually perpendicular lines of spots in the Laue photograph are $\{100\}_{\alpha}$ and $\{100\}_{\beta}$ planes and we denote the plane with the smaller (larger) number of $x$-ray spots as $\{100\}_{\alpha}\left(\{100\}_{\beta}\right)$. While the dHvA frequencies observed in the two types of planes were different for a certain set of frequencies, ${ }^{11}$ we make no further reference to the two types of $\{100\}$ planes in the present paper. This is because we are interested mainly in determining the state of compensation, the presence of open orbits, and their directions; to describe these properties it is not necessary to keep the full point group symmetry in mind for this particular structure.

${ }^{18}$ L. M. Falicov and P. R. Sievert, Phys. Rev. Letters 12, 558 (1964); Phys. Rev. 138, A88 (1965).

${ }^{19}$ R. W. Stark and L. M. Falicov, in Progress in Low Temperature Physics, edited by C. J. Gorter (North-Holland Publishing Co., Amsterdam, 1967), Vol. V, p. 235.

${ }_{20}$ R. W. G. Wyckoff, Crystal Structures (John Wiley \& Sons, Inc., New York, 1963), 2nd ed., Vol. I, p. 346.

${ }^{21} \mathrm{~J}$. Ahn, Ph.D. thesis, Massachusetts Institute of Technology, 1968 (unpublished).
TABLE II. Sample properties.

\begin{tabular}{cccc}
\hline \hline Identification & $\begin{array}{c}\text { Sample } \\
\text { axis }^{\mathbf{a}}\end{array}$ & $\begin{array}{c}\text { Residual } \\
\text { resistance } \\
\text { ratio }^{\mathbf{b}}\end{array}$ & $\begin{array}{c}\text { Method } \\
\text { of growth }^{\text {B }}\end{array}$ \\
\hline I-A & $\langle 100\rangle$ & 190 & Bridgman \\
I-B & $\langle 100\rangle$ & 286 & Bridgman \\
II-A & $\langle 110\rangle$ & 240 & Bridgman \\
II-B & $\langle 110\rangle$ & 400 & Bridgman \\
III-A & $\langle 111\rangle$ & 315 & Czochralski \\
III-B & $\langle 111\rangle$ & 316 & Bridgman \\
IV- & $\hat{\imath}_{n \mathbf{s}^{\mathbf{c}}}$ & 520 & Bridgman \\
\hline \hline
\end{tabular}

a Sample axis is parallel to current density $\mathrm{J}$.

b Estimated error $\sim 5 \%$.

- Unit vector in nonsymmetry direction which is $26.5^{\circ}, 27.5^{\circ}$, and $29.0^{\circ}$ from $\langle 100\rangle,\langle 110\rangle$, and $\langle 111\rangle$, respectively.

\section{B. Preparation of Samples}

The phase diagram of the Au-Sb system ${ }^{22}$ shows that the $\mathrm{AuSb}_{2}$ phase has a very limited range of homogeneity. While this phase is shown in the phase diagram as a vertical line implying infinitesimal width, the exact limits of the phase are not known. The nature of the phase diagram also makes it clear that for crystal growth from the melt, an excess of antimony in the melt should be avoided if single-crystal $\mathrm{AuSb}_{2}$ is to precipitate on cooling. This circumstance plus the rather high vapor pressure of antimony at the melting temperature of gold ( $\sim 10$ Torr) were important factors for which allowance had to be made to obtain high-purity crystals, i.e., crystals with nearly exact stoichiometry.

Single-crystal ingots were grown either by the Bridgman or Czochralski methods. The starting materials were " 59 "-grade $\mathrm{Au}$ and $\mathrm{Sb}$ shot..23 Use of "69"-grade materials did not improve the crystalline perfection in terms of the residual resistance ratio (RRR) [RRR

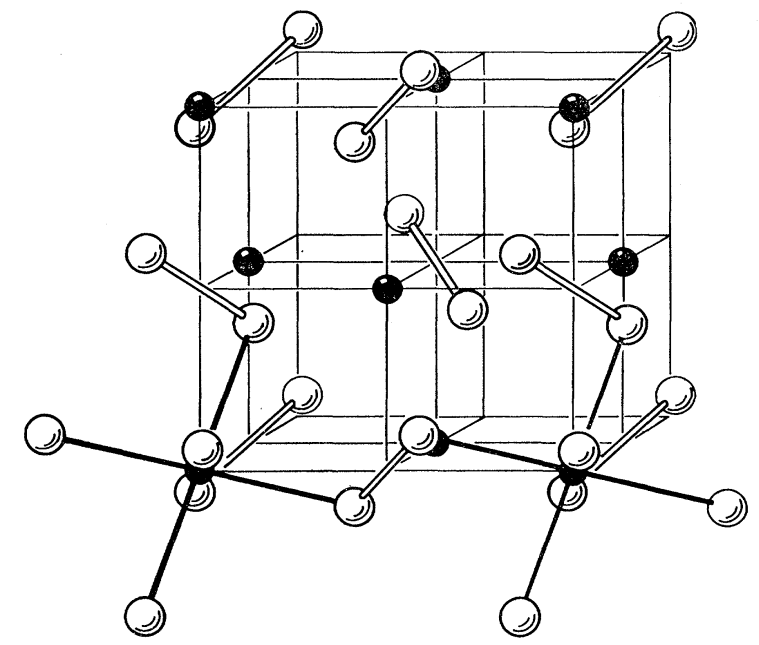

FIG. 1. Crystal structure of $\mathrm{AuSb}_{2}$. Black spheres represent $\mathrm{Au}$ atoms and white spheres Sb atoms.

${ }^{22}$ M. Hansen, Constitution of Binary Alloys (McGraw-Hill Book Co., New York, 1958), p. 230.

${ }^{23}$ Purchased from Cominco American Inc., Spokane, Wash. 


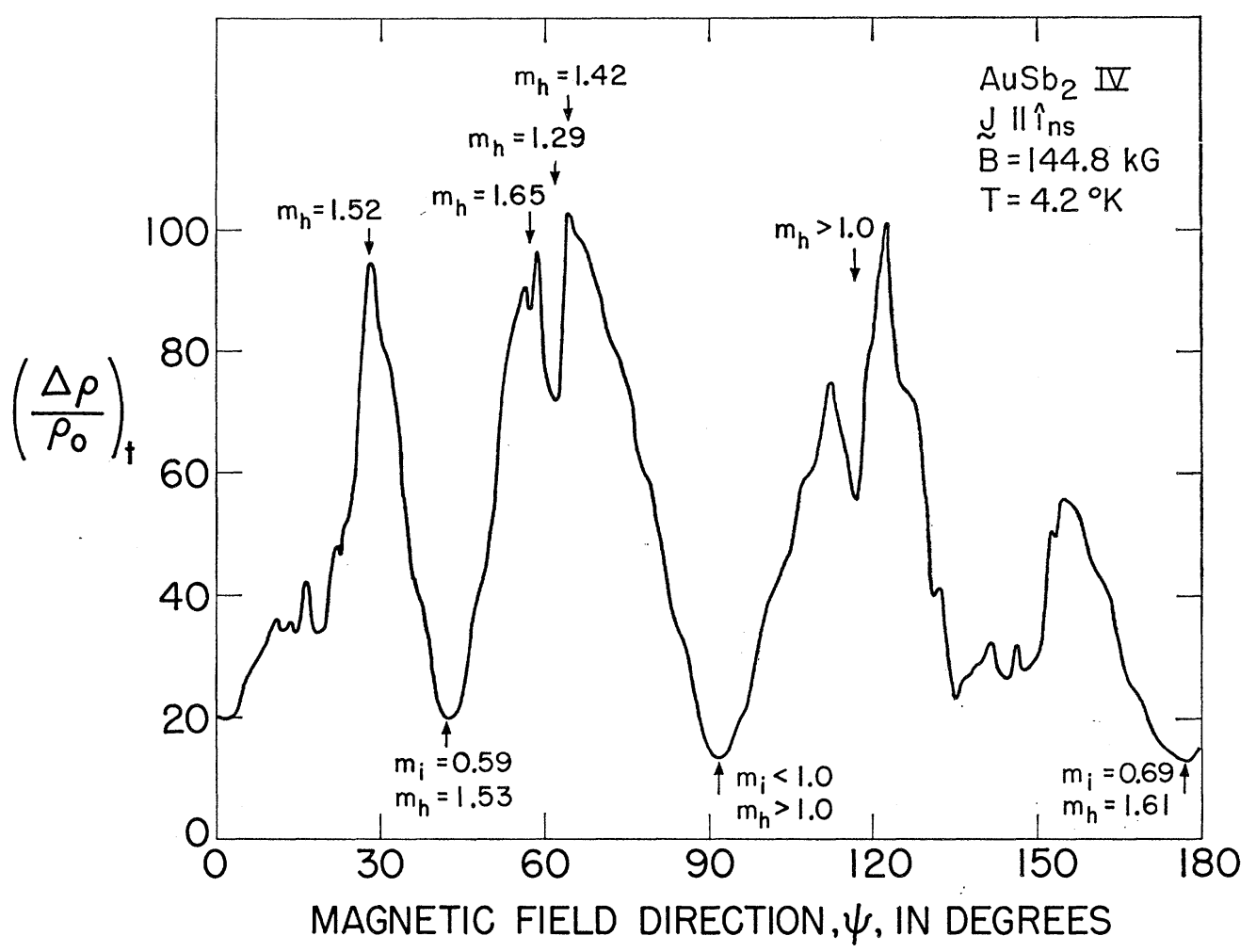

FIG. 2. Transverse MR anisotropy of the nonsymmetry sample, AuSb 2 IV at $144.8 \mathrm{kG}$. The numbers $m_{i}$ and $m_{h}$ are fielddependence exponents as explained in the text. The field dependence at $\psi=28^{\circ}, 40^{\circ}, 92^{\circ}$, and $177^{\circ}$ is shown in Fig. 3 .

$\left.=\rho\left(295^{\circ} \mathrm{K}\right) / \rho\left(4.2^{\circ} \mathrm{K}\right)\right]$. This means that the $\mathrm{RRR}$ is more dependent on other crystalline imperfections than the impurities from the starting materials.

In the Bridgman method, ingots were grown in a split graphite mold with a pointed end in a zone-melting apparatus. ${ }^{24}$ Typically, the growing rate was about $2.5 \mathrm{~cm} / \mathrm{h}$. The single-crystal ingots grown were cylinders of dimensions $0.8 \times 5.0 \mathrm{~cm}$. The initially solidified end of the ingot always had the highest RRR ( 500-600) but was usually polycrystalline near the tip. The other end of the ingot had the lowest RRR ( 150-250). In order to save the portion of the ingot with the highest RRR, the polycrystalline tip was successively removed with a spark cutter ${ }^{25}$ until Laue photographs ensured that the remaining portion was truly a single crystal. The Czochralski method was tried in an attempt to increase the RRR above about 500 . A cylindrical seed of dimension $0.5 \times 1.3 \mathrm{~cm}$ with a $\langle 111\rangle$ orientation was spark cut from a Bridgman crystal. The Czochralski crystal, grown in a conventional fashion, was a cylindrical shape with dimension $1.3 \times 2.0 \mathrm{~cm}$. However, the RRR was uniformly about 315 .

Samples for MR experiments were shaped by spark cutting. First, the single-crystal ingot was oriented by $\mathrm{x}$-rays in a goniometer for the desired current axis direction to $\pm 1^{\circ}$. Then a cylindrical sample (1.5${ }^{24}$ Model Z-82, Materials Research Corp., Orangeburg, N. Y.
25 "Servomet," Metals Research Ltd., Cambridge, England.
$2.5 \mathrm{~mm}$ in diameter, $0.8 \mathrm{~cm}$ long) along the oriented axis was spark-cut using a copper tube as a cutting tool. Two flat mutually perpendicular sides along the cylinder axis were spark-planed so that one flat face could be used to solder voltage leads and the other flat face could be used to glue the specimen to the sample holder with a known orientation. The final dimensions of the MR samples were $0.15 \times 0.8 \mathrm{~cm}$. The orientations and identification of all the MR samples along with the RRR values are summarized in Table II.

\section{Measurement Technique}

All experimental results were obtained in a $5.1-\mathrm{cm}$ bore, water-cooled solenoid providing a maximum field of $150 \mathrm{kG}$. The experiments were done at temperatures between 1.2 and $4.2^{\circ} \mathrm{K}$ with the temperature regulated below $4.2^{\circ} \mathrm{K}$ by a Walker regulator. ${ }^{26}$ The pressure was monitored with a Wallace-Tiernan aneroid gauge for the pressure range of $50 \leq p \leq 760 \mathrm{~mm} \mathrm{Hg}$ and by a Stokes-McLeod gauge for the range $1 \leq p \leq 50$ $\mathrm{mm} \mathrm{Hg}$. The experiment consisted of determining the MR and Hall voltages as a function of crystal orientation and field magnitude. The sample holder and electronic apparatus used in this work has been described previously. ${ }^{2,27}$ As described previously, ${ }^{4} \mathrm{Hall}$ effect mea-

${ }^{26}$ E. J. Walker, Rev. Sci. Instr. 30, 834 (1959).

${ }^{27}$ D. J. Sellmyer, Rev. Sci. Instr. 38, 434 (1967). 

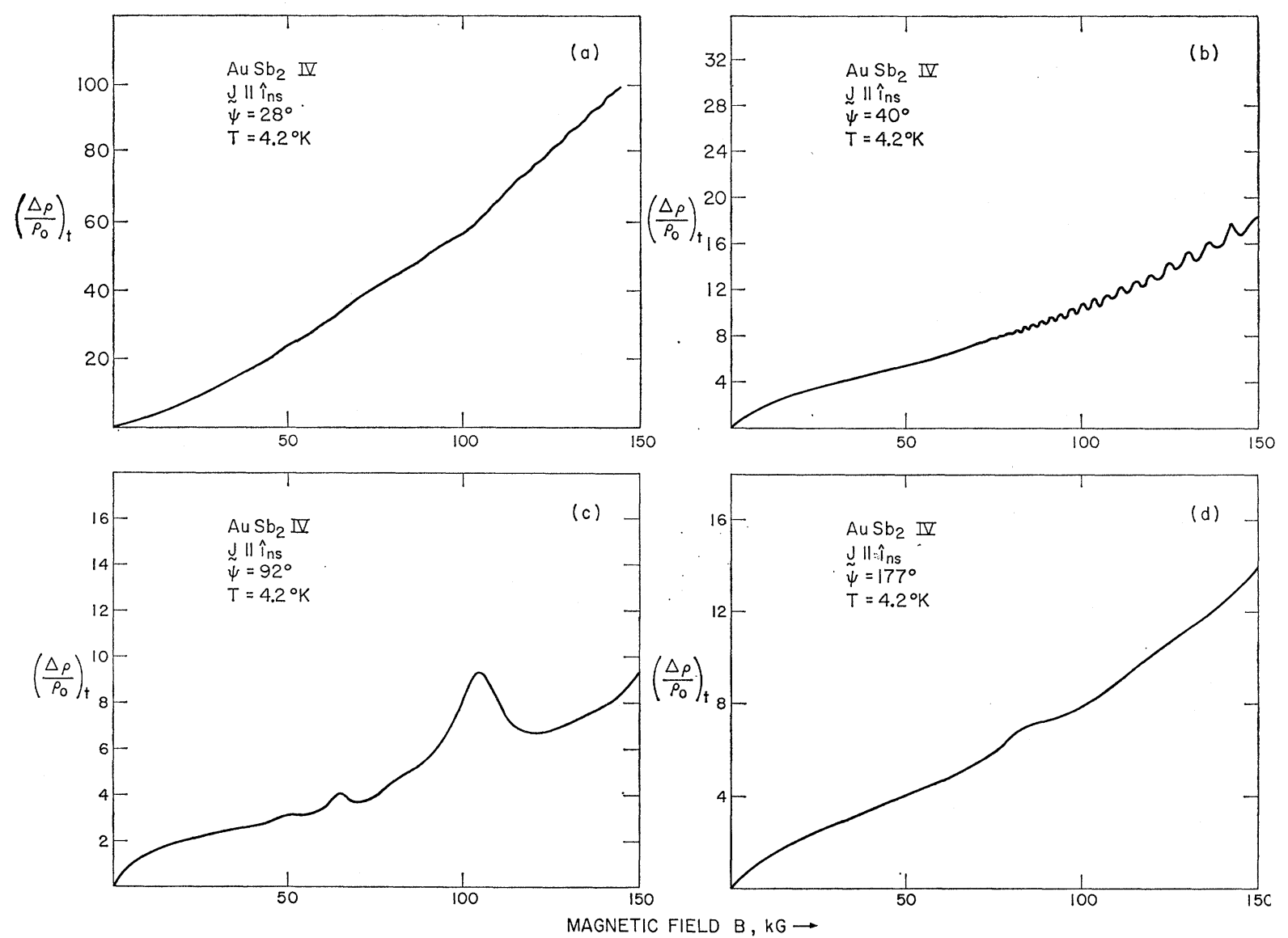

FIG. 3. Field dependence of the MR at various positions of Fig. 2. (a) shows the approach to quadratic behavior at a maximum of Fig. 2. (b)-(d) show inflection behavior in the symmetry planes $\{110\},\{110\}$, and $\{100\}$, respectively.

surements were made at several high-symmetry directions. The standard procedure of reversing both the field and current directions was followed so that the Hall voltages were obtained by combining four measured voltages.

The MR voltage, $V(B)$ is directly related to the MR

$$
\Delta \rho / \rho_{0} \equiv\left[\rho(B)-\rho_{0}\right] / \rho_{0}=[V(B)-V(O)] / V(O),
$$

where $V(O)$ and $\rho_{0}$ are the zero-field voltage and resistivity, respectively. In the MR plots of Sec. IV, transverse MR $(B \perp \mathbf{J})$ is denoted by $\left(\Delta \rho / \rho_{0}\right)_{t}$ and longitudinal MR $(\mathbf{B} \mid \mathbf{J})$ by $\left(\Delta \rho / \rho_{0}\right)_{l}$.

\section{RESULTS}

The magnetoresistance results were obtained from samples having their axes along $\langle 100\rangle,\langle 110\rangle,\langle 111\rangle$, and a nonsymmetry direction (sample IV; Table II). Selected transverse-rotation and field-dependence plots from the nonsymmetry sample and the $\langle 110\rangle$ and $\langle 111\rangle$ samples are shown in Figs. 2-6.

In order to apply the theory summarized in Table I, it is necessary that the high-field condition is at least close to being satisfied. A very rough estimate of $\left\langle\omega_{c} \tau\right\rangle_{\text {av }}$ can be obtained from a simple two-band model in which it is assumed that the densities and mobilities of the electrons and holes are equal. According to this model, the $\mathrm{MR}$ is given by ${ }^{15}$

$$
\left(\Delta \rho / \rho_{0}\right)_{t}=\left(\omega_{c} \tau\right)^{2} .
$$

From Fig. 2, the maximum value of $\left(\Delta \rho / \rho_{0}\right)_{t}$ is $\simeq 100$, which gives $\omega_{c} \tau \simeq 10$ at $150 \mathrm{kG}$. On the basis of this simple model, then, the high-field condition is satisfied in the highest fields used in this investigation. Furthermore, the theory predicts that the longitudinal MR saturates in the high-field limit for any state of compensation and for any open-orbit configuration. ${ }^{15}$ Figure 5(c) shows a typical example of the field dependence of the longitudinal MR. The nearly complete saturation with $\left(\Delta \rho / \rho_{0}\right)_{l} \simeq 1$ at fields of the order of $50 \mathrm{kG}$ and higher indicates that the high-field requirement is satisfied reasonably well.

Since the distinguishing feature of the MR behavior is either quadratic growth or saturation with fields, function-of-field plots were made at the angles $\psi$ corresponding to the extrema of the MR in Fig. 2. Such plots for sample IV are shown in Fig. 3. When $\psi=28^{\circ}$, Fig. 3(a), which corresponds to a maximum in Fig. 2, 


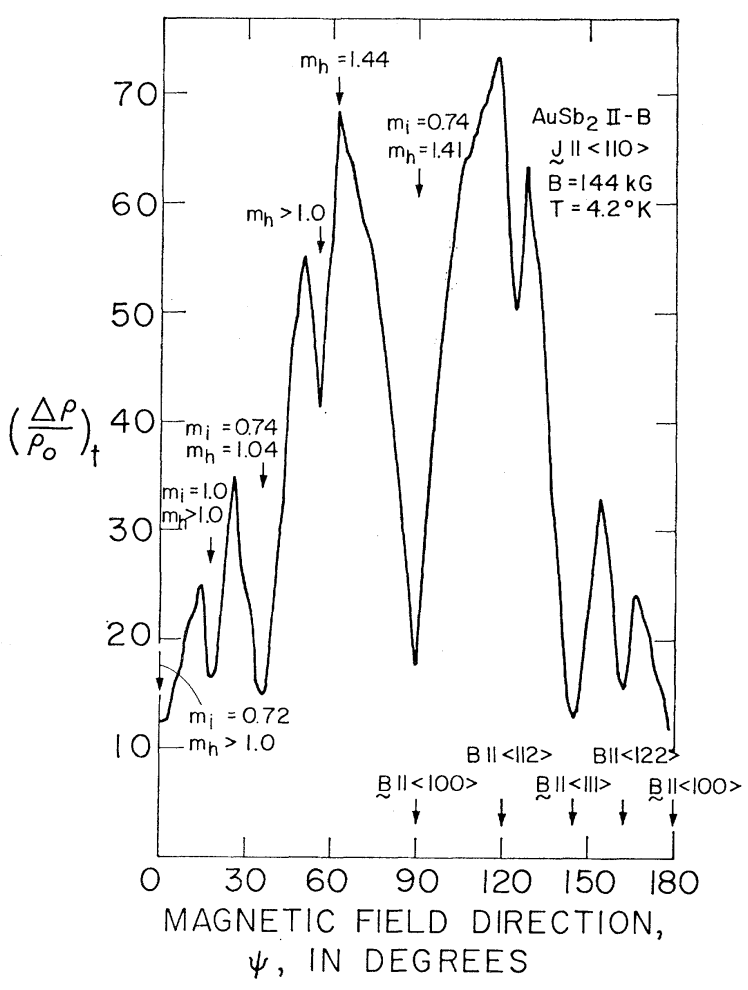

FIG. 4. Transverse MR anisotropy of $\mathrm{AuSb}_{2}$ II-B sample in a $\{110\}$ plane. Positions of major MR minima are indicated along the dashed line in Fig. 7.

the MR grows faster than linearly with field. At other angles, Figs. 3(b)-3(d), which correspond to minima in Fig. 2, the MR tends toward saturation at intermediate fields but grows much faster at higher fields. This behavior, which appears often in $\mathrm{AuSb}_{2}$ (see Figs. 5 and 6), is referred to loosely as an inflection in the MR. Additional features of the field dependence of the MR are the ubiquitous quantum oscillations, sometimes quite large, which are superimposed on the steady MR. The frequencies of the oscillations in Figs. 3(c), 5(a), and $5(\mathrm{~d})$ are of the order $10^{5} \mathrm{G}$ while those seen in Figs. 3(b) and 5(b) are about $2 \times 10^{6} \mathrm{G}$. The orientation dependence of these frequencies is discussed separately in II.

The field dependence of the monotonic part of the $\mathrm{MR}$ is expressed as

$$
\Delta \rho / \rho_{0}=a B^{m_{i, h}},
$$

where $a$ is a constant, the exponent $m_{h}$ is to be evaluated at the highest fields, and $m_{i}$ is to be evaluated at the intermediate fields below the inflection only if it occurs. Log-log plots of MR versus field gave the exponents and these values are shown in Fig. 2 at the corresponding extrema. In some cases, e. g., Fig. 3(c), the exponents and the points of inflection could not be accurately determined either due to a very mild inflection or largeamplitude low-frequency MR oscillations. In such cases, inequality signs, as determined by visual inspection, are used to indicate the qualitative field dependence.

In order to investigate the MR behavior over portions of the whole stereogram, constant-field rotation plots were made at various tip angles $\phi$. This result for the nonsymmetry sample is shown on a stereogram in Fig. 7, which shows the loci of MR minima in the rotation plots. The lower-left quarter of this figure also shows $\{100\},\{110\}$, and $\{112\}$ planes as well as various crystallographic directions for reference. It is seen from Fig. 7 that the angles at which the MR exhibit maxima in Fig. 2 correspond to general field directions. Since the MR is large for general field directions and the MR minima occur only when the field lies in a symmetry plane or is close to a high-symmetry axis, we conclude that $\mathrm{AuSb}_{2}$ is a compensated metal. This conclusion is consistent with the rules ${ }^{2}$ for compensation in metallic compounds since the total number of electrons per primitive cell is an even number. Figure 7 shows that the loci of the MR minima lie in the (011), (011), (110), (001), and (112) planes and the angles between the directions normal to these planes (i.e., poles) and $\mathbf{J}$ are nearly $90^{\circ}$. Thus, in accordance with Table $I$ and the field dependences of the MR at these minima [Figs. $3(\mathrm{~b})-3(\mathrm{~d})]$, we conclude that there exist periodic open orbits in $\langle 100\rangle,\langle 110\rangle$, and $\langle 112\rangle$ directions at fields lower than about $50 \mathrm{kG}$. An important point is that, at these minima, the tendency toward saturation at intermediate fields is replaced by a tendency toward quadratic behavior in the highest fields (see Fig. 2). Specific examples of the inflection behavior associated with $\langle 110\rangle$ and $\langle 112\rangle$ open orbits are shown in curves I and II, respectively, of Fig. 6; these curves were obtained under ideal geometric conditions, viz., $\gamma=90^{\circ}$. The inflection behavior will be discussed further in Sec. $V$.

While dips in the rotation plots have been observed in planes other than $\{100\},\{110\}$, and $\{112\}$, no inflection behavior was found at these dips and the exponent $m$ was always greater than 1 . It is possible that in the limit of extremely pure samples and large fields $\left(\left\langle\omega_{c} \tau\right\rangle_{\mathrm{av}} \rightarrow \infty\right)$ some of these higher-order planes also would show saturating behavior. However, as has been shown in the case of copper ${ }^{28}$ and $\mathrm{AuGa}_{2},{ }^{4}$ such higherorder open orbits are often the result of various combinations of open orbits using "necks" in the low-index directions such as $\langle 100\rangle,\langle 110\rangle$, and $\langle 111\rangle$. The higherorder open orbits do not contribute significantly, therefore, to the qualitative topological conclusions that may be drawn about the Fermi surface. In $\mathrm{AuSb}_{2}$, the $\langle 100\rangle$, $\langle 110\rangle$, and $\langle 112\rangle$ open orbits are the strongest open orbits observed and no doubt include the primary openorbit directions.

The $\langle 112\rangle$-directed open orbits do not appear to exist for all field directions in $\{112\}$ planes. This was

${ }^{28}$ J. R. Klauder, W. A. Reed, G. F. Brennert, and J. E. Kunzler, Phys. Rev. 141, 592 (1966). 

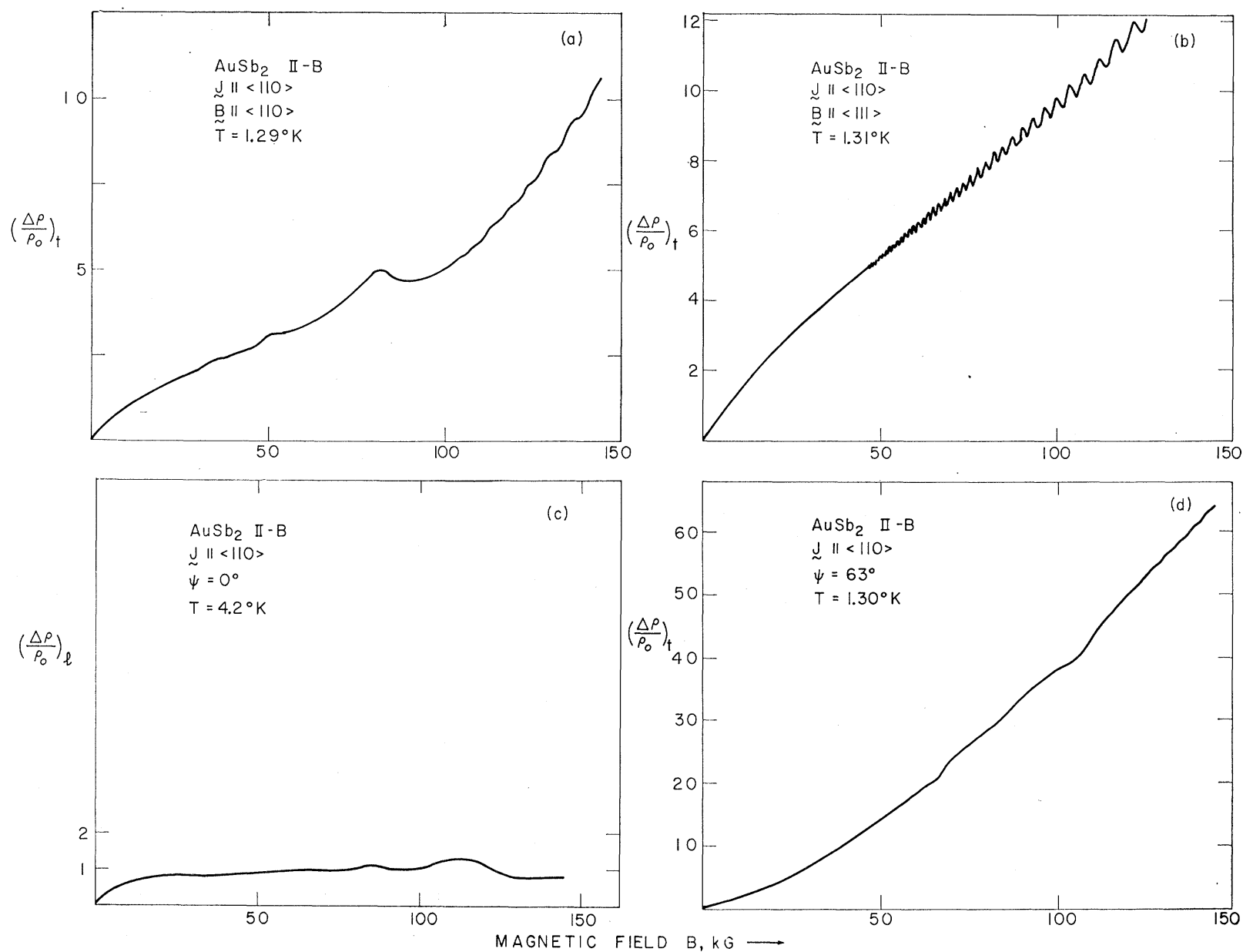

FIG. 5. Field dependence of MR of AuSb 2 II-B sample: (a) $\mathrm{B} \|\langle 110\rangle$; (b) $\mathbf{B} \|\langle 111\rangle$; (c) $\mathrm{J}\|\mathrm{B}\|\langle 110\rangle$; (d) $\psi=63^{\circ}$ on Fig. 4 .

shown as follows: Referring to Fig. 8, the $\mathrm{J} \|\langle 110\rangle$ sample was successively tipped away from the (110) plane by a small amount and a rotation plot was made at each $\phi$ position. This made it possible to trace the

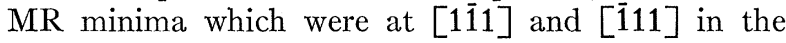
transverse geometry [B in (110), see Fig. $4, \psi \simeq 35^{\circ}$, $145^{\circ}$ ]. By determining the tip angle at which the minima disappeared in the rotation plot, the arc length of the one-dimensional region away from $\langle 111\rangle$ as shown in Fig. 8 was found to be $27^{\circ}$. This is only a rough estimate, because when $\phi \neq 0$, the MR contains a longitudinal component as well as the usual transverse component so the dips tend to be washed out. However, it has been shown ${ }^{6,29}$ that the characteristic indication of open orbits in the transverse MR, viz., pronounced extrema, is retained in the nontransverse geometry as long as $\phi$ is not too large. Another important point is that only two segments of the $\langle 112\rangle$ open orbits were detected in the field region covered in Fig. 8. These two segments of $\{112\}$ have their plane normals directed

${ }^{29}$ W. A. Reed, E. Fawcett, and R. R. Soden, Phys. Rev. 41, 354 (1961); 139, A1557 (1965). toward [ $\overline{1} 12]$ and [112] and these directions are both perpendicular to $\mathbf{J} \|[110]$, i.e., $\gamma=90^{\circ}$. All other "potential" minima in the MR did not appear because $\gamma$ is very much different from $90^{\circ}$. This confirms the point mentioned in Sec. II A that open orbits are easily missed in a compensated metal if the experimental geometry is such that $\gamma$ is not close to $90^{\circ}$.

We have approximately determined the two-dimensional regions of open orbits. Various methods for such determination in compensated metals are described by Alekseevskii and Gaidukov, ${ }^{30}$ and Schirber. ${ }^{31}$ Our procedure was to measure the MR in $1.5^{\circ}$ intervals near $\{100\}$ and $\{110\}$ planes with $\mathrm{J} \|\langle 100\rangle$ and $\langle 110\rangle$ samples, respectively. The inflections characteristic of $\mathrm{B} \|\langle 100\rangle,\langle 110\rangle$, and $\langle 111\rangle$ [e.g., see Figs. 5(a) and 5(b)] were lost as the field was rotated away from these directions. Taking the angular regions in which the MR inflection persisted as the two-dimensional regions of open orbits, the angular limits of these regions are as

${ }^{30}$ N. E. Alekseevskii and Y. P. Gaidukov, Zh Eksperim. i Teor. Fiz. [English transl.: Soviet Phys.-JETP 14, 256 (1962)].

31 J. E. Schirber, Phys. Rev. 131, 2459 (1963). 


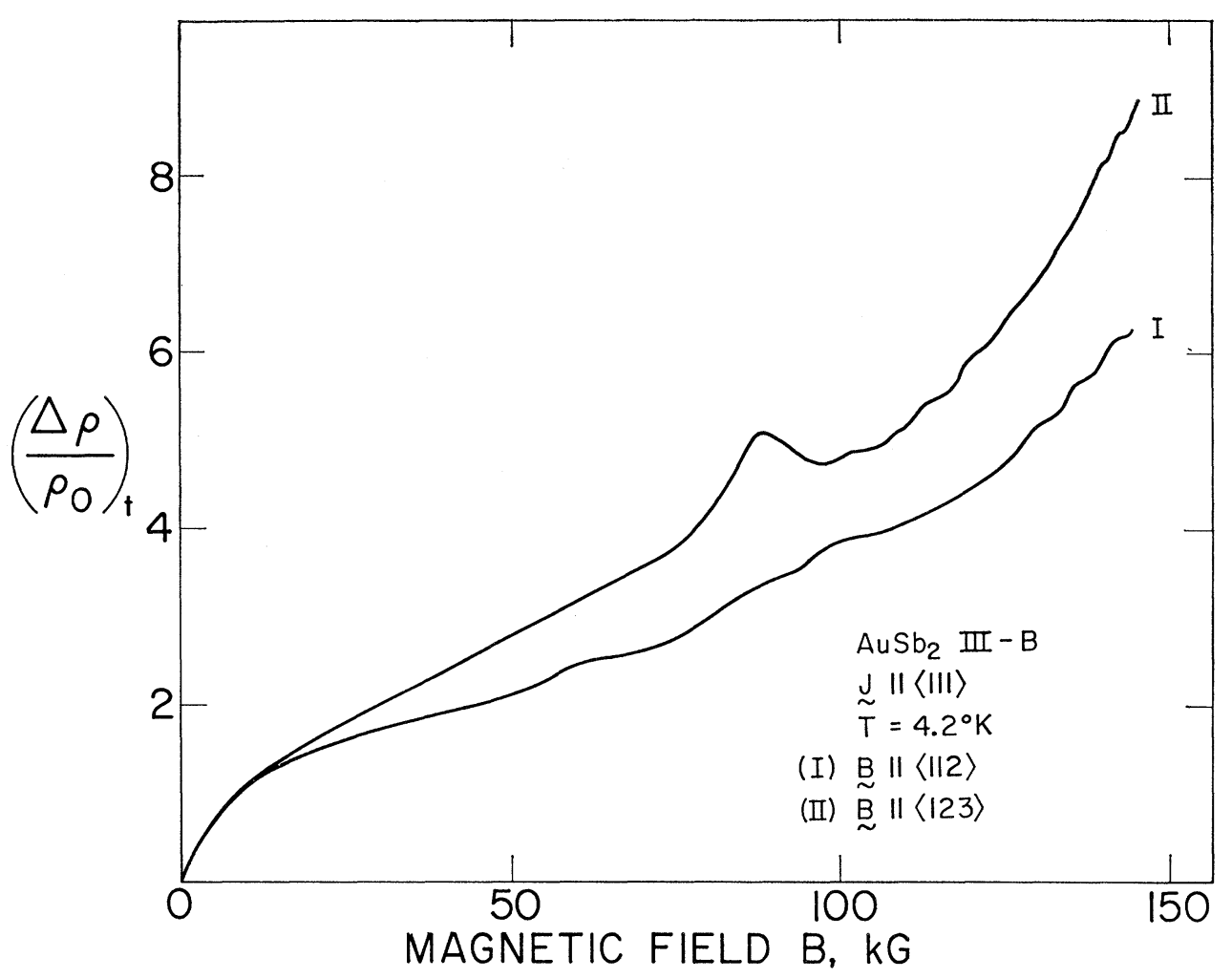

Fig. 6. Field dependence of MR of AuSb 2 III-B sample: curve I, B $\|\langle 112\rangle$ showing behavior of $\langle 110\rangle$ open orbit; curve II, B $\|\langle 123\rangle$ showing behavior of $\langle 112\rangle$ open orbit.

follows :

$$
\begin{aligned}
& 2^{\circ} \text { from }\langle 100\rangle \text { near }\{100\}, \\
& 3^{\circ} \text { from }\langle 100\rangle \text { near }\{110\}, \\
& 2^{\circ} \text { from }\langle 110\rangle \text { near }\{100\}, \\
& 6^{\circ} \text { from }\langle 110\rangle \text { near }\{110\}, \\
& 6^{\circ} \text { from }\langle 111\rangle \text { near }\{110\} .
\end{aligned}
$$

Although the field was rotated near one-dimensional regions of open orbits, the field could not have been exactly in these planes because, if this had been the case, no open orbits could have been detected since $\gamma=0$ (Table I). Further evidence for this is shown by the slight asymmetry of the transverse rotation plot of Fig. 4 about $\psi=90^{\circ}$. Therefore, the approximate size of the two-dimensional regions of open orbits is as shown in Fig. 8 (Sec. IV of the stereogram).

Although the sample with $\mathbf{J}$ parallel to a nonsymmetry direction $\left(\mathrm{AuSb}_{2} \mathrm{IV}\right)$ proved to be the most informative with regard to open orbit directions, it was necessary to show that the MR data obtained from $\mathrm{J} \|\langle 100\rangle,\langle 110\rangle$, and $\langle 111\rangle$ samples was consistent with the above open-orbit topology. Data obtained from all of these samples were tabulated and used, as discussed in Sec. $\mathrm{V}$, to construct a self-consistent interpretation of the MR data at all fields and field directions used in this investigation.
Hall coefficients $R_{H}$ were measured at high-symmetry directions for sample II B $(\mathbf{J} \|\langle 110\rangle)$. The voltages on the Hall probes contained large quantum oscillations as a function of field so that, at times, it was not wholly obvious how to obtain the monotonic part of these voltages. However, the Hall voltages obtained from these plots were roughly proportional to $B$ and the resulting slopes gave values for $R_{H}$ of $-18.2,-2.12$, and $-1.85 \times 10^{-5} \mathrm{~cm}^{3} / \mathrm{C}$ for $\mathrm{B} \|\langle 100\rangle,\langle 110\rangle$, and $\langle 111\rangle$ field directions, respectively. However, for reasons to be discussed below, it is not clear that any of these values are related directly to geometrical features of the Fermi surface.

\section{DISCUSSION}

\section{A. Magnetoresistance}

In the present section we wish to discuss more fully the field dependence of the magnetoresistance. Inflection behavior in the MR has been observed in rhenium ${ }^{29}$ and $\mathrm{Zn}^{32}$ but $\mathrm{AuSb}_{2}$ is apparently the only metal in which it has been observed for all field directions in three different crystallographic planes which are onedimensional open-orbit regions. The inflection behavior observed in the MR of rhenium was shown to result from essentially two groups of carriers, one having an

${ }^{32}$ W. A. Reed and G. F. Brennert, Phys. Rev. 130, 565 (1963). 


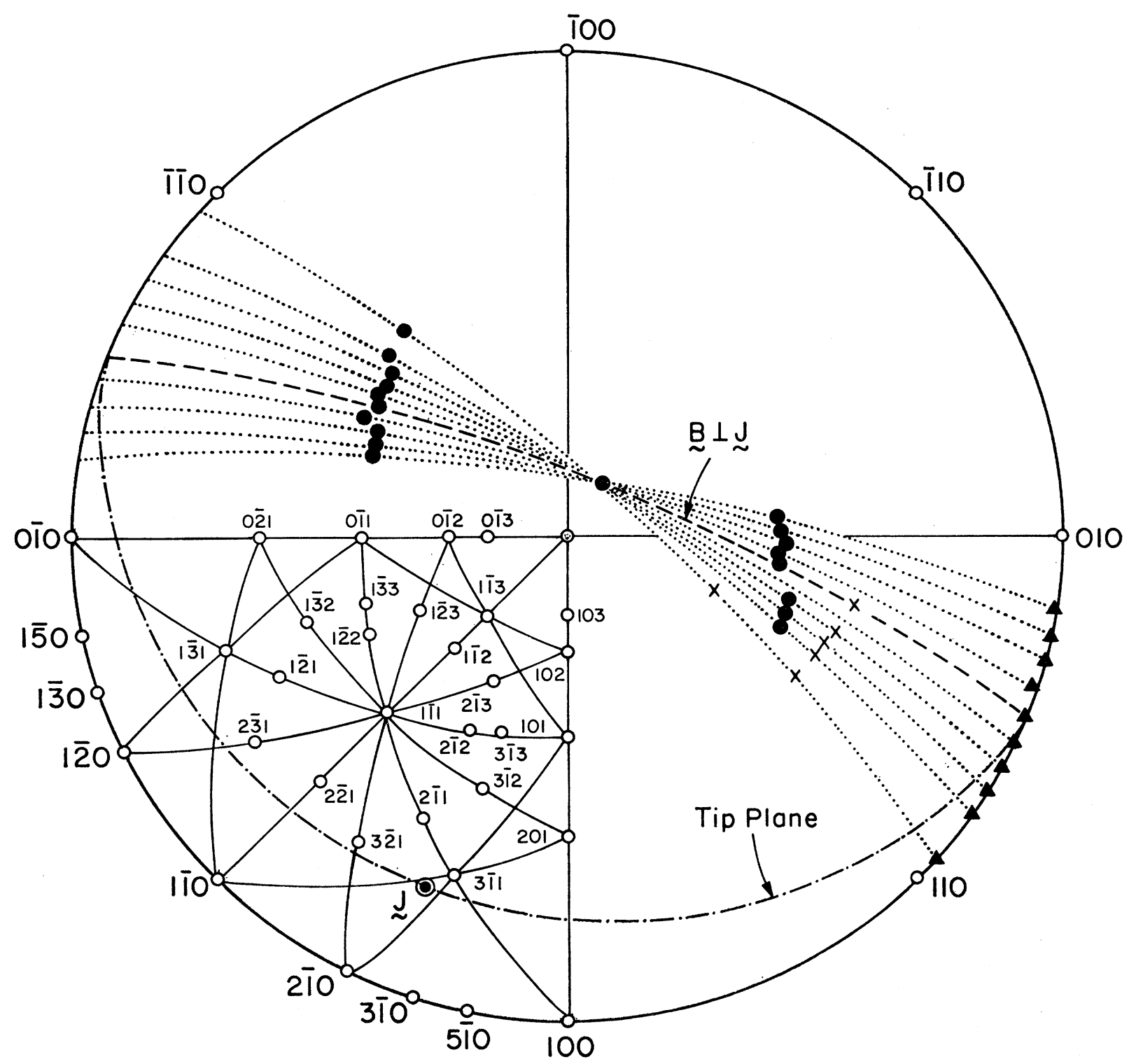

- $\underset{\sim}{B}$ in $\{110\}$

$\triangle \underset{\sim}{B}$ in $\{100\}$

$x \underset{\sim}{B}$ in $\{112\}$

FIG. 7. Stereogram showing positions of MR minima of $\mathrm{AuSb}_{2}$-IV sample. The MR minima in transverse rotation (the great circle perpendicular to J) were obtained from Fig. 2. Other MR minima were obtained from nontransverse rotations. The lower-left quarter also shows $\{100\},\{110\}$, and $\{112\}$ planes as well as various crystallographic directions for reference.

order of magnitude higher mobility than the other. ${ }^{29}$ In what follows, we argue that our results cannot be explained by a similar hypothesis but can be explained in terms of magnetic breakdown of the open orbits into closed orbits at intermediate and high fields $(B \gtrsim 50 \mathrm{kG})$.

It has been shown in Sec. IV that the essential features of the MR are as follows:
(1) With B in a nonsymmetry plane the MR always tends toward quadratic behavior and there is never an inflection behavior. While the closest approach to quadratic behavior has been $m \simeq 1.7$ for a general field direction, it seems reasonable that with somewhat higher-purity samples the theoretically expected value of $m=2.0$ would be observed; similar deviations of $m$ 


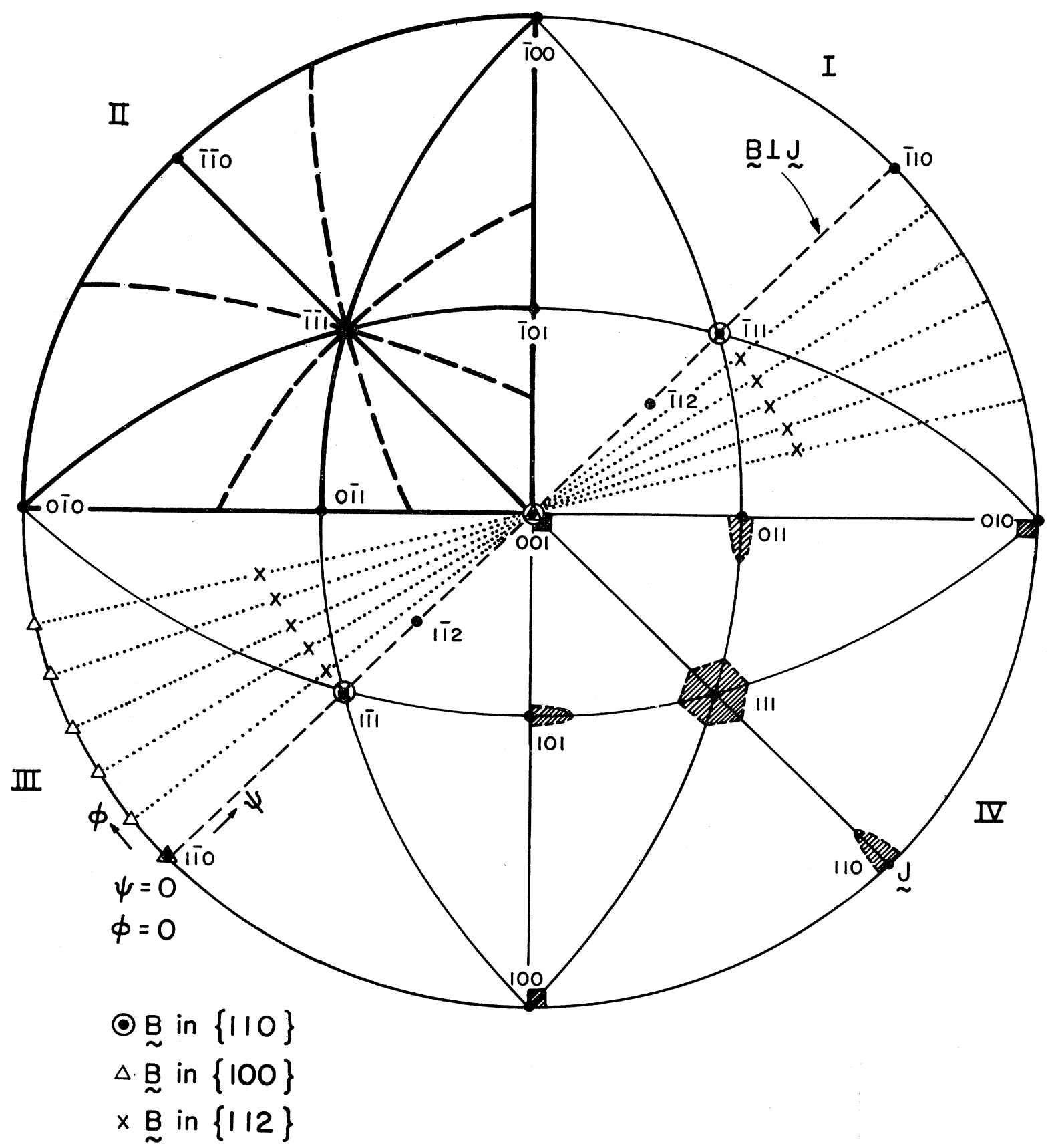

FIG. 8. Stereogram showing: (a) the $\{100\}$ and $\{110\rangle$ planes (heavy lines) and $\{112\}$ planes (dashed lines) in quadrant II; (b) locus of points exhibiting MR dips caused by open orbits in the $\{100\}$ and $\{112\}$ planes in quadrants I and III; and (c) the field directions for which aperiodic open orbits exist-shaded regions in quadrant IV.

to values slightly less than 2 have been observed in many other elemental metals and metallic compounds with resistance ratios between 100 and $1000 .^{2,4,33}$

(2) When $B$ is in $\{100\},\{110\}$, and in portions of $\{112\}$ planes and when $\gamma$ is close to $90^{\circ}$ there is always a tendency toward saturation at intermediate fields

${ }^{33}$ W. A. Reed and E. Fawcett, Phys. Rev. 136, A422 (1964).
( $\sim 30-50 \mathrm{kG}$ ) and a tendency toward quadratic be havior in the highest fields. We wish to consider whether this behavior could be explained by a model having (at least) two bands of equal electron and hole densities but having mobilities differing by an order of magnitude. If we denote the effective fields of the high and low mobility hands by $\left(\omega_{c} \tau\right)_{1}$ and $\left(\omega_{c} \tau\right)_{2}$, respectively, then we can define the intermediate $(\mathrm{I})$ and high $(\mathrm{H})$ 
field regions by the inequalities

$$
\begin{array}{lll}
\left(\omega_{c} \tau\right)_{1} \gg 1 & \text { and } & \left(\omega_{c} \tau\right)_{2}<1 \text { in region } I, \\
\left(\omega_{c} \tau\right)_{2} \gg 1 & \text { and } & \left(\omega_{c} \tau\right)_{2} \gg 1 \text { in region } H .
\end{array}
$$

In field region I, the low-mobility band would not affect the MR significantly and the metal would behave as an uncompensated metal. By considering various field directions and various possible open- and closed-orbit combinations, it is easily shown that this model can give rise, under certain conditions, to a saturating to quadratic transition between regions I and II; however, it cannot give this behavior when the current direction is normal to the open-orbit direction as is observed in $\mathrm{AuSb}_{2}$. It therefore appears that the saturating behavior observed at intermediate fields is truly the result of open orbits rather than some of the carriers not being in the high-field region.

One might inquire also whether the inflection behavior could be explained in terms of a small error in the orientation of current axes of the crystals. The equation for the MR is ${ }^{15}$

$$
\Delta \rho / \rho=a+b B^{2} \cos ^{2} \gamma,
$$

where $b$ might be large and $\gamma=90^{\circ}+\epsilon$ where $\epsilon$ is small. At low fields $\Delta \rho / \rho \sim a$, but at high fields the second term would become larger than the first and $\Delta \rho / \rho \sim B^{2}$. However, it is easily shown using experimental values of the MR to obtain an upper bound on $b$ and a reasonable value for $a$, that one would require an $\epsilon$ of $\sim 20^{\circ}$ to explain the inflection behavior in this way.

A more reasonable explanation of the saturating-toquadratic transition is that of magnetic breakdown. This is strongly suggested by the observation that, as seen in Figs. 3, 5, and 6, the transition from saturating to quadratic behavior is always accompanied by rather large quantum oscillations in the MR. For example, the ratio of oscillation amplitude to steady $\mathrm{MR}, \delta \sigma / \sigma$, is as much as $\sim 40 \%$ [Fig. 3(c)]. It is unlikely that these oscillations are ordinary de Haas-Shubnikov (dHS) oscillations such as are observed in the semimetals $\mathrm{Bi}^{34}$ and $\mathrm{Sb}^{35}$ It is shown in II that $\mathrm{AuSb}_{2}$ is not a semimetal but has rather large pieces of Fermi surface as well as some small pockets. It is in this sense a "good" metal and it has been observed that quantum oscillations in good metals for which $\delta \sigma / \sigma$ is of order unity can be explained only on the basis of an oscillatory probability for breakdown. ${ }^{36}$ Such large-amplitude oscillations have been observed in $\mathrm{Zn},{ }^{36} \mathrm{Mg},{ }^{19}$ and $\mathrm{Sn},{ }^{37}$ and we defer further discussion of the orientation, temperature, and field dependence of these oscillations to II. With regard to the monotonic variation of the MR, a

${ }^{34}$ C. G. Grenier, J. M. Reynolds, and J. R. Sybert, Phys. Rev. 132,58 (1963).

${ }^{35}$ G. N. Rao, W. H. Zebouni, C. G. Grenier, and J. M. Reynolds, Phys. Rev. 133, A141 (1964)

${ }^{36}$ R. W. Stark, Phys. Rev. Letters 9, 482 (1962).

${ }^{37}$ R. C. Young, Phys. Rev. 152, 659 (1966). possible model involving magnetic breakdown which can explain the observed MR behavior is that of Falicov and Sievert. ${ }^{18}$ Figure 5 of their paper shows the result of a semiclassical model which predicts a change from a saturating to a quadratic MR behavior as breakdown changes the orbits from open orbits to closed orbits with compensation.

Referring to Fig. 6, curve II shows large oscillatory "humps" near $90 \mathrm{kG}$ whose amplitude is about $15 \%$ of the steady MR. Both curves I and II (curve I for $\langle 110\rangle$ open orbit and curve II for $\langle 112\rangle$ open orbit) tend to saturation but the MR inflections develop near $90 \mathrm{kG}$. Thus, the $\langle 112\rangle$ open orbit $(\mathbf{J} \|\langle 111\rangle$ and $\mathbf{B} \|\langle 123\rangle$ and the $\langle 110\rangle$ open orbit $(\mathrm{J} \|\langle 111\rangle$ and $\mathbf{B} \|\langle 112\rangle)$, which exist up to $\sim 90 \mathrm{kG}$, are lost by breakdown at highest fields. The same conclusion hold for the $\langle 100\rangle$ open orbits as is shown, for example, in Fig. 3(d).

Further consideration of the results obtained with the "high-symmetry" samples (I, II, and III) shows that all of the MR behavior when $B \|\langle 100\rangle,\langle 110\rangle$, and $\langle 111\rangle$ can be explained by the type-V (Table I) behavior in the intermediate-field region and by the typeII behavior in the highest fields as a consequence of magnetic breakdown.

There is an interesting implication regarding the band structure of $\mathrm{AuSb}_{2}$ in the above interpretation of the loss of open orbits by magnetic breakdown. This is that there seem to be many rather small energy gaps separating bands which contain pieces of the Fermi surface. We may make a rough estimate of some of the gaps involved in the open-orbit trajectories as follows. There is an upturn in the MR indicating loss of open orbits between 50 and $100 \mathrm{kG}$. If we therefore assume a characteristic breakdown field $B_{c} \simeq 75 \mathrm{kG}$ for all three types of open orbits, we may use Eq. (2) to obtain an estimate of the energy gap $\boldsymbol{\epsilon}_{g}$. Using NFE values appropriate for $\mathrm{AuSb}_{2}$, viz., $K=\frac{1}{4} \pi$ and $\epsilon_{F}=10.5 \mathrm{eV}$, it is found that $\epsilon_{g} \simeq 85 \mathrm{meV}$. It is interesting that this very rough estimate agrees rather well with the weak band gaps of $\sim 80 \mathrm{meV}$ calculated on the basis of the low-field galvanomatic behavior in Ref. $9 .{ }^{38} \mathrm{Also}$, it is surprising that all three types of open orbits apparently involve gaps of about the same size and we know of no other pure metal or compond which exhibits similar behavior.

\section{B. Hall Effect}

As shown by Table I and explained in Ref. 15, it is possible to obtain geometric information about the Fermi surface from Hall data at singular-field directions. It is natural to ask whether the $\langle 100\rangle,\langle 110\rangle$, and $\langle 111\rangle$ directions are singular-field directions in $\mathrm{AuSb}_{2}$, since they are at the intersections of one-dimensional regions of open orbits. It is necessary that the $\langle 111\rangle$ directions be singular-field directions because of the threefold rotation symmetry about these axes. The $\langle 100\rangle$ and

${ }^{38}$ P. R. Emtage (private communication). 
$\langle 110\rangle$ axes need not be singular-field axes on grounds of symmetry but it is easy to imagine open sheets of $\langle 100\rangle$ directed pipes, consistent with the crystal symmetry, which would, for example, give a two-dimensional open-orbit region about $\langle 100\rangle$, while the $\langle 100\rangle$ direction itself would not be a singular-field direction. In any case, if a high-symmetry axis is a singular-field direction, $\Delta \rho / \rho \propto B^{0}$, the Hall voltage $V_{H} \propto B$ and $V_{H}$ measures $\Delta n$ (Table I) independent of the direction of $\mathrm{J}$ (except that $\mathbf{J}$ must be perpendicular to $\mathrm{B}$ ).

Now we have never observed a clear saturation of the MR for B parallel to any of the three directions in question. There are always inflections with $m_{i}$ slightly less than 1 and $m_{h}$ slightly greater than 1 . Further, there are often very large oscillations in the MR for $B \gtrsim 70 \mathrm{kG}$, so, as explained above, it seems likely that magnetic breakdown occurs at fields of the order of 50 $\mathrm{kG}$ and higher. Under these conditions it is not clear that a measurement of the Hall coefficient, $R_{H}$, will give meaningful values for $\Delta n$.

Nevertheless, measurements of $R_{H}$ were made for $\mathrm{B} \|\langle 100\rangle,\langle 110\rangle$, and $\langle 111\rangle$ directions and the values of $\Delta n$ (Table I) obtained were $-0.68,-5.87$, and -6.70 , respectively. Now it is clear that $|\Delta n|$ cannot be larger than 2.0 at a singular-field direction. ${ }^{15}$ Thus the equivocal behavior of the MR and the unphysical value of $\Delta n$ for $\mathrm{B} \|\langle 111\rangle$, which should be a singular-field direction, lends further support to the breakdown hypothesis in this direction. Similarly, at $\langle 110\rangle|\Delta n|>2$ suggesting either that it is not a singular-field direction or that magnetic breakdown exists there also. The only field directions which give reasonable values for $\Delta n$ are the $\langle 100\rangle$ directions. It is possible to calculate an openorbit neck dimension with this value but in view of the lack of definite saturation of the MR at these directions, we do not feel that such a calculation is warranted.

\section{SUMMARY}

The results of this investigation of the high-field magnetoresistance of $\mathrm{AuSb}_{2}$ indicate that it is a compensated metal in agreement with its even number of electrons per primitive cell. The Fermi surface of $\mathrm{AuSb}_{2}$ supports open trajectories in $\langle 100\rangle,\langle 110\rangle$, and $\langle 112\rangle$ directions. The field dependence of the magnetoresistance associated with these open orbits exhibited a transition from a saturating behavior at intermediate fields $(\lesssim 50 \mathrm{kG})$ to a tendency toward quadratic behavior for fields above $100 \mathrm{kG}$. It was argued that this behavior could not be explained by assuming that there was one group of carriers which did not achieve the high-field condition until $B \lesssim 100 \mathrm{kG}$. However, the observed behavior was shown to be consistent with magnetic breakdown of the open orbits to closed orbits and a return to the compensated state in the highest fields. Of course, this latter interpretation cannot be true except in a qualitative sense because even at $150 \mathrm{kG}$, the magnetic breakdown is not truly complete.

\section{ACKNOWLEDGMENTS}

The authors would like to acknowledge the interest and support of Professor B. L. Averbach. They thank Professor A. F. Witt, C. J. Herman, and E. H. Backman for their help in crystal growth, and P. P. Kelleher for the construction of parts of the apparatus. Support of this research by the Advanced Research Projects Agency through the Center for Materials Science and Engineering of M.I.T. is gratefully acknowledged. 